\title{
Experimental Investigation of Axisymmetric Transitional Shock Wave Boundary Layer Interactions at Mach 5
}

\author{
E. Erdem ${ }^{1}$, K. Kontis ${ }^{1}$, E. Johnstone ${ }^{2}$, N. Murray $^{3}$, and J. Steelant ${ }^{3}$
}

\section{Introduction}

When shock waves interact with boundary layers developing on solid surfaces Shock Wave Boundary Layer Interactions (SWBLIs) form, which embed a series of complicated flow phenomena such as flow separations, shear layers, transition mechanisms, shock-shock interactions, etc. SWBLI can induce separation which causes loss of a control surface effectiveness, drop of an air intake efficiency and it may be the origin of large scale fluctuations such as air-intake buzz, buffeting or fluctuating side loads in separated propulsive nozzles. The subsequent reattachment of the separated shear layer on a nearby surface gives rise to local heat transfer rates which can be far in excess of those of an attached boundary layer [1]. Because of their dramatic importance, SWBLIs have been extensively studied during the past 50 years and are still the subject of active research due to their complexity and difficulty to predict their behaviour especially in transitional/turbulent regime $[2,3,4]$. Most of the work done in this field over the years has been focussed on fully developed turbulent flows since most practical applications were at transonic and low supersonic speeds at altitudes where Reynolds numbers are large and turbulent boundary layer flows are encountered. With the decrease of altitude, there is a rise in Reynolds number so that transition, which first occurs far downstream penetrates into the SWBLI region. Therefore transitional SWBLIs, in which the incoming boundary layer is in a transitional state (natural transition), or in which transition is induced within the interaction itself (triggered transition), appear to be even more unsteady and could have greater adverse effects, and yet have received little attention [5]. For hypersonic SWBLIs only a few studies are available such as the ones from Boyce and Hillier [6] and Murray et al. [7]. These studies involved axisymmetric SWBLI configurations, avoiding three dimensional side effects. The test model used was a hollow axisymmetric centrebody on which natural transition took place and the impinging shock was localised on turbulent boundary layer. Obtained pressure and heat transfer distributions assessed the severity of the interaction in terms of the associated peaks and oil flow visualisation demonstrated Goertler type vortices at the reattachment. Present experimental study focusses on this problem with an impinging shock wave including both transition mechanisms; natural and triggered transition. The methodology involves high-speed wind tunnel testing at Mach

AeroPhysics Lab, School of MACE, University of Manchester, Manchester, M13 9PL, UK · Fluid Gravity Engineering, The Old Coach House, 1 West Street, Emsworth, PO10 7DX, UK · European Space Research and Technology Centre, Postbus 299, Keplerlaan 1, 2200 AG Noordwijk, The Netherlands 
5 with an axisymmetric hollow model similar to studies of Boyce and Hillier [6] and Murray et al. [7]. The associated flow diagnostics include; conventional stagnation and wall pressure measurements, schlieren visualisation, Quantitative Infrared Thermography (QIRT) and Pressure Sensitive Paints (PSP).

\section{Experimental Methodology}

The experiments documented herein were performed in High SuperSonic Tunnel (HSST) in Aero-Physics Laboratory of the University of Manchester. The tunnel is of the intermediate blowdown(pressure-vacuum) type which uses dry air as the working fluid and is shown schematically in Fig. 1 (top). The various components of this facility have been discussed in detail elsewhere $[8,9]$. The stagnation pressure can range from 5 to 8 bar and thereby unit Reynolds numbers, $R e / m$, of between $4-16 \cdot 10^{6} 1 / m$ can be achieved. The variations in Mach number and unit Reynolds number were found to be $\pm 0.4 \%$ and $\pm 3.7 \%$ respectively [8, 9]. A hollow centrebody model, made of PEEK, is used for the experimental campaign. The model is $50.8 \mathrm{~mm}$ in diameter and $450 \mathrm{~mm}$ in length with a wall thickness of $3 \mathrm{~mm}$ as shown in Fig. 1 (bottom left) and is painted with matt black paint to increase emissivity. In terms of the shock generator to create SWBLI, an axisymmetric cowl model, made of aluminium, is used. The flow deflection introduced by the cowl is 7 degrees from inside as shown in Fig. 1 (bottom right). Stagnation and wall static pressure

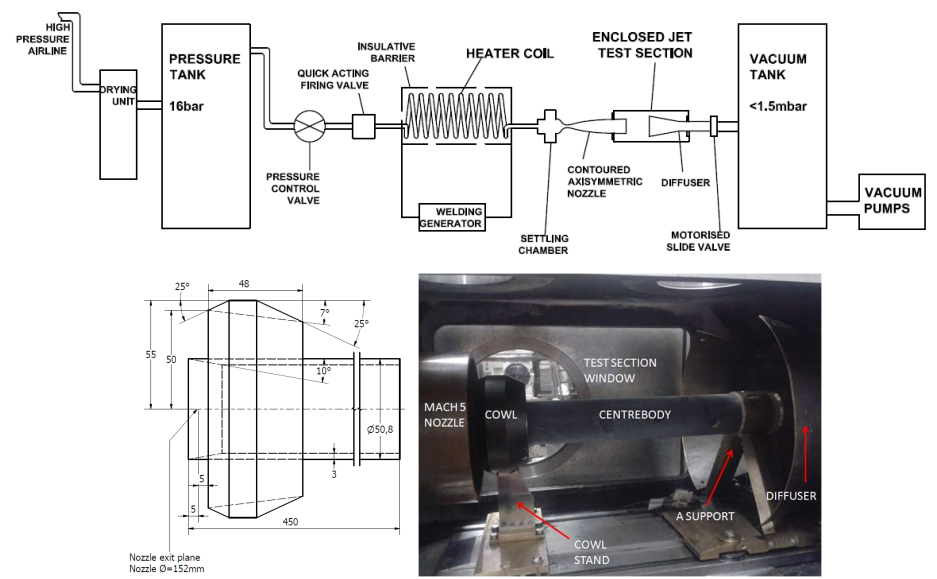

Fig. 1 Top: University of Manchester HSST schematic layout, bottom left: technical drawing of cowl and its starting position (SWBLI@90mm)with respect to the centrebody, bottom right: test section of HSST with the centrebody and cowl.

measurements are done using high-sensitivity Kulite $(\mathcal{C})$ pressure transducers. A pitot probe (3 $\mathrm{mm}$ in diameter) attached to a Kulite pressure transducer (XTE-190M, 100 psi range) via small chamber and a K-type thermocouple probe ( $3 \mathrm{~mm}$ in diameter) with a junction diameter of $1.0 \mathrm{~mm}$ are located at the settling chamber to measure $p_{0}$ and $T_{0}$ respectively. In case of wall pressure measurements, 12 pressure tappings (of $1.5 \mathrm{~mm}$ outer diameter) are located on the model; the first three tappings are offset by 20 degrees with respect to the centreline whereas the others are located at 
the centreline. Analog signals from all the sensors are acquired at $5 \mathrm{Khz}$ by a highspeed Data Acquisition (DAQ) card, National Instruments(NI) (c) PCI-6251, after they are conditioned by SXCI-1000 unit [8, 9]. Toepler's z-type schlieren technique is adapted for flow visualisation that consist of a continuous light source of Palflash 501 (Pulse Photonics) with a focusing lens and a $1 \mathrm{~mm}$ wide slit, two $203.2 \mathrm{~mm}$ inches parabolic mirrors with $1828.8 \mathrm{~mm}$ focal length, a knife edge, a set of Hoya $49 \mathrm{~mm}$ close-up lenses and a digital Canon SLR camera, EOS-450D, 12MP. The camera is set to have a long shutter and the light source is set to spark mode (pulse duration $0.75 \mu \mathrm{sec}$ to resolve flow features with sharpness). The layout of the optical setup and the DAQ architecture with measurement chain for conventional pressure and temperature measurements is shown in Fig. 2. The change of surface temperature distribution is measured using infrared camera (FLIR SC655), which has a temperature range of $-20^{\circ} \mathrm{C}$ to $150^{\circ} \mathrm{C}$ with accuracy of $\pm 1 \%$. The spectral range of the camera is between 7.5 and $13 \mu \mathrm{m}$. It is installed on the top of the test section and views the model obliquely at 25 degrees with respect to vertical through a Germanium window with transmission range of 3 to $12 \mu \mathrm{m}$. The surface temperature is recorded in the entire test duration at $100 \mathrm{~Hz}$ at $640 \times 240$ pixel $^{2}$ resolution. Figure 2 shows the setup for QIRT measurements. The pressure sensitive paint is composed of Ruthenium, Ethanol, MTEOS and $\mathrm{HCl}$ and sprayed on top of the white base coat applied on the centrebody. This PSP is a polymer based paint and has superior pressure sensitivity, however it has a slower response time compared to porous PSP. For the excitation of PSP, a pair of light emitting diode (LED) panels with a peak wavelength of $470 \mathrm{~nm}$ is used. Each LED panel is comprised of $13 \times 10$ LED arrays. The LED panel is placed on each side of test section. The main advantage of placing two LED panels, as shown in Fig. 2, on both sides of test section is that it can provide a uniform illumination on the surface of model. The centrebody is rotated 90 deg to have the same area as in QIRT tests assuming the cowl is perfectly axisymmetric. A 12 bit LaVision Image Intense CCD camera with $1280 \times 1040$ pixel $^{2}$ resolution is used for emission acquisition at 9 fps with $10 \mathrm{~ms}$ exposure time. The wavelength emitted from Platinum based pressure sensitive paint is found to be around $610 \mathrm{~nm}$ using a spectrometer. Different wavelength filters are required to separate the emission from the illumination. Based on the spectral analysis, a combination of orange $550 \mathrm{~nm}$ long pass and an IR rejection filter are employed in front of the CCD camera. The former allows the transmission of light with $\lambda>550 \mathrm{~nm}$ while the latter rejects light with wavelength longer than $700 \mathrm{~nm}$.

\section{Results}

The results of the experimental campaign include schlieren photography, QIRT, PSP and wall pressure measurements. Figure 3 shows spark schlieren visualisation of the flowfield with different SWBLI positions during the time-averaging window specified above. The horizontal axis is shifted with respect to SWBLI location, i.e. SWBLI location becomes zero $\left(x-x_{S W B L I}\right)$. Following structures can be observed clearly; centrebody leading edge shock (only visualised in SWBLI@90mm case), impinging cowl shock, cowl expansion fan and the shear layer at the cowl trailing edge, upstream separation region (upstream of the shock impingement location) 


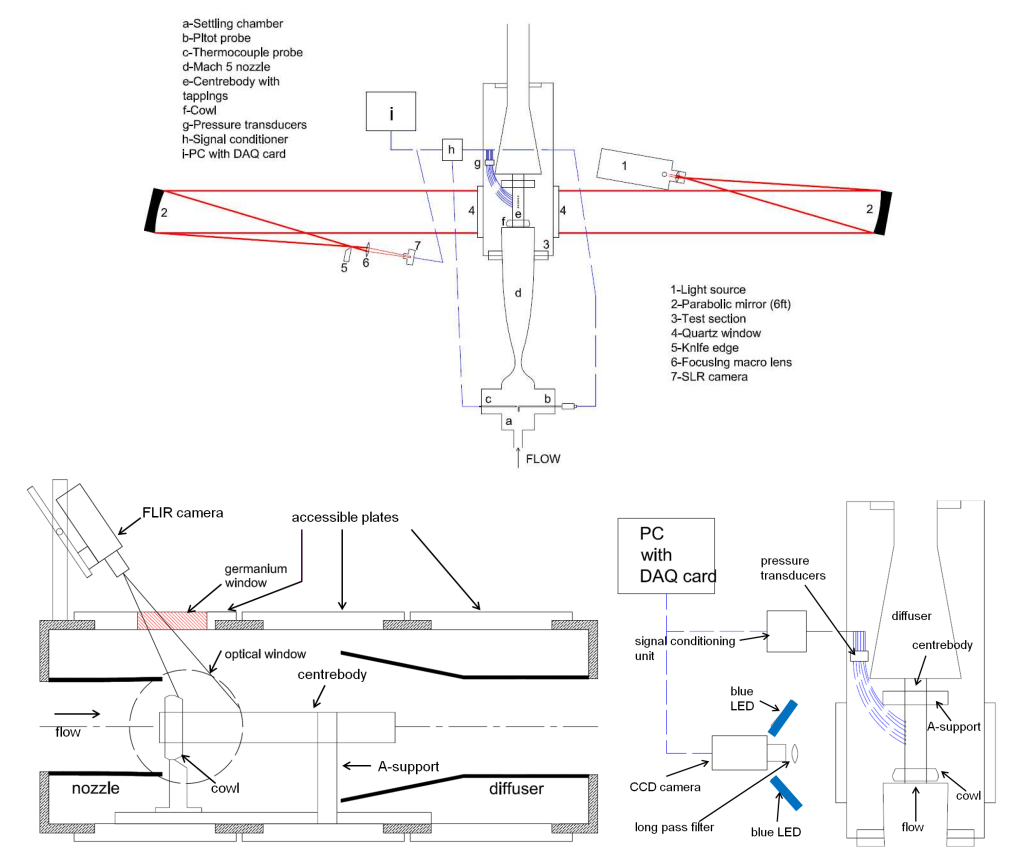

Fig. 2 Schematic setup of schlieren visualisation with DAQ architecture [8,9], Schematic setup of QIRT measurements, Schematic setup of PSP measurements..

with the separation shock, expansion wave on top of the separation region, the reattachment shock, and finally the nozzle shock. In the downstream of the SWBLI region the boundary layer structures are oscillatory and the turbulent in nature. In general as the intermittency is increased boundary layer becomes more turbulent and skin friction coefficient rises. Hence it becomes more resistant to adverse pressure gradient imposed by the impinging shock, which would result a relatively smaller SWBLI region. Nevertheless due to blockage in optical access owing to cowl, the upstream extent of SWBLI region can not be shown.

Figure 4 (top left) show Stanton number profiles over the centreline of the centrebody for all the cases. For each case, i.e.SWBLI@90, 190, 200, 220 and 255mm, the data from the tests without the cowl are also plotted. In addition theoretical laminar and turbulent distributions of heat flux and Stanton number over a flat plate using reference temperature method are included. The agreement between the experiments and the theory is good in terms of laminar and turbulent heat flux distributions. The transition region is found to be broad, starting from around $150 \mathrm{~mm}$ and ending nearly at $300 \mathrm{~mm}$. Figure 4 (top right) show azimuthal Stanton number distributions on max heat flux lines. SWBLI@90mm shows oscillatory behaviour around the circumference, the reason for that might be the presence of streamwise vortices. SWBLI@190 and 200mm cases also exhibit oscillatory behaviour but smaller in peaks and troughs.SWBLI@200mm case is very similar to SWBLI@190mm case in terms of heat flux distribution. Figure 4 (bottom left) shows the shifted Stanton 


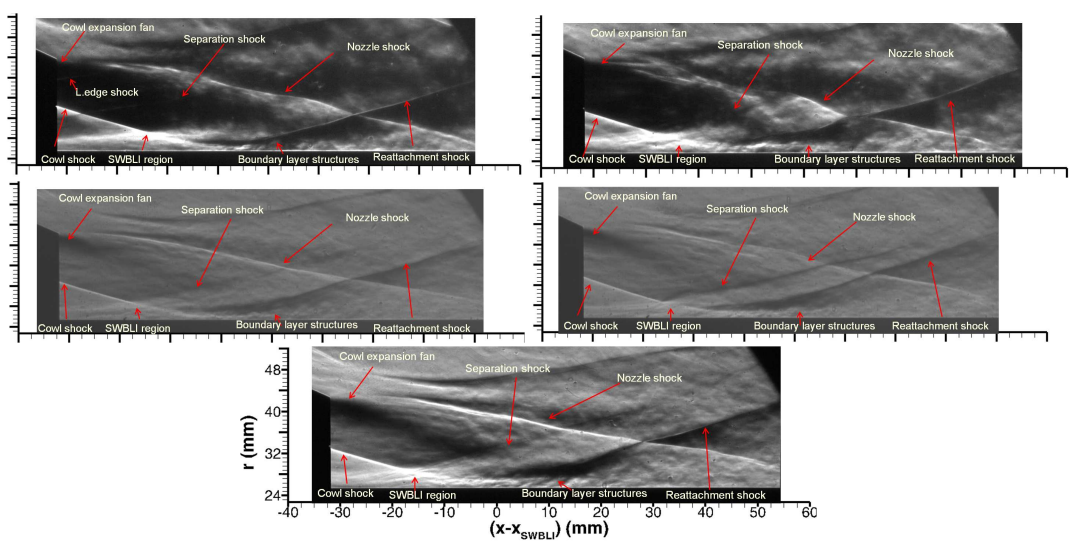

Fig. 3 Schlieren visualisation of the flowfield at the interaction region for SWBLI@90mm, SWBLI@190mm,SWBLI@200mm, SWBLI@220mm and SWBLI@255mm cases in descending order.

number distributions over centreline of the centrebody. The horizontal axis is shifted with respect to SWBLI location, i.e. SWBLI location becomes zero. The heat flux rise due to the SWBLI is overlapping on top of each other for SWBLI@190, 200, 220 and 255 cases up to $15 \mathrm{~mm}$ upstream of inviscid SWBLI position, afterwards it starts to deviate. The rise is more aggressive for SWBLI@190 and 200mm cases compared to SWBLI@220 and 255mm cases. Downstream of heat transfer peak profiles start to overlap around $20 \mathrm{~mm}$ downstream of the origin. Smaller rise in profiles at around $50 \mathrm{~mm}$ downstream of origin is due to the influence of the nozzle shock. This overlapping behaviour clearly demonstrates the quality and repeatability of the measurements. SWBLI@90mm case has a bigger heat flux peak and steeper rise due to the fact that the boundary layer is undergoing transition to turbulence (triggered transition) throughout the SWBLI region. Figure 4 (bottom right) also shows the azimuthally averaged Stanton number values (between -45 and 45 degree azimuthal angles) at maximum heat flux lines against Reynolds number based on inviscid position of the impinging shock. The error bars do not represent the experimental uncertainties but manifest the variation of heat flux across the circumference between -45 to 45 degrees (from min to max). Nominally laminar values are found to be biggest and the highest intermittency values are the smallest. This finding is consistent with the findings from Vandomme et al. [5]. The uncertainties in the heat flux distributions are found to be around $9 \%$.

Figure 5 (left) shows shifted non-dimensional wall pressure distributions over the centrebody. The horizontal axis is shifted with respect to inviscid SWBLI location, i.e. SWBLI location becomes zero. The pressure rise due to the SWBLI is ovelapping on top of each other for SWBLI@190, 200, 220 and 255mm cases. SWBLI@90mm case has a steeper rise on the other hand. However due to the discrete nature of the tappings, the peak of the pressure rise for SWBLI@90mm case can not be observed. The maximum non-dimensional pressure value is found to be 

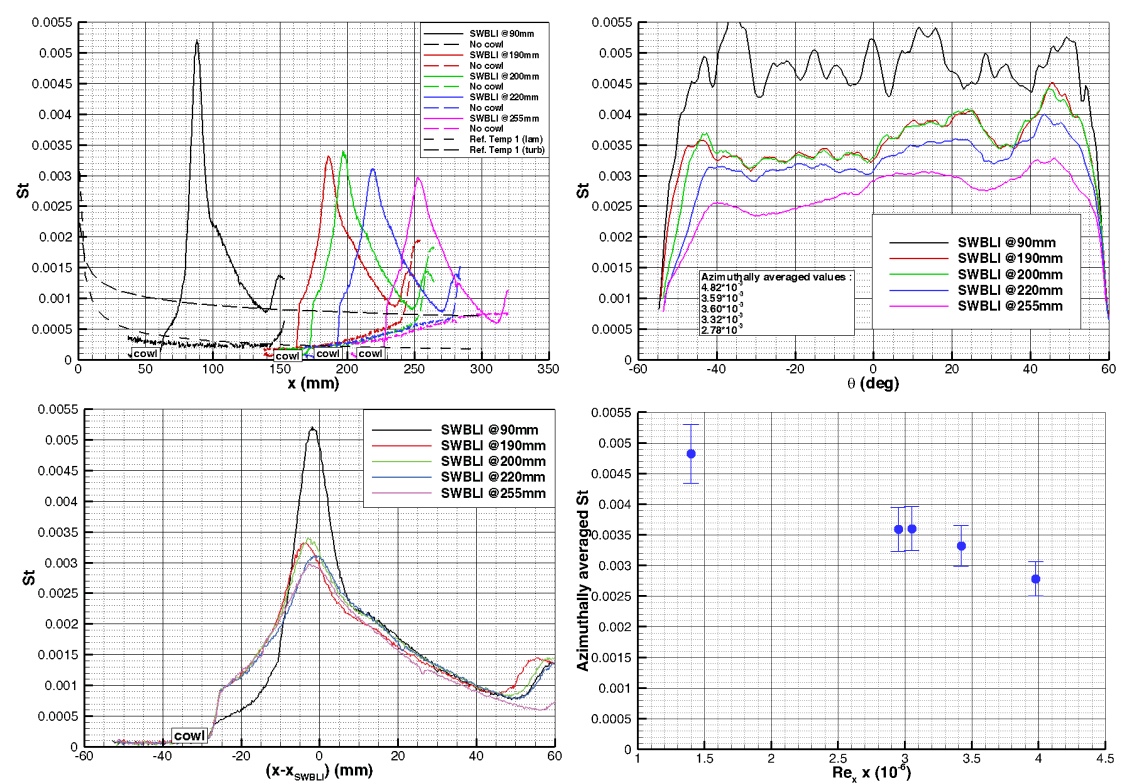

Fig. 4 Top left: centreline Stanton number distributions for all cases with theoretical laminar and turbulent estimates, Top right: azimuthal Stanton number distributions at maximum heat flux lines for all cases, bottom left: centreline Stanton number distributions over the centreline of the centrebody shifted with respect to impinging shock location; bottom right: azimuthally averaged Stanton number values at maximum q lines vs. Reynolds number for all cases.

around 5.8. This value is found to be greater than the value of 4.55 found using double regular reflection of 7 degrees. The non-dimensional pressure values are found to be accurate within $4 \%$. Figure 5 (right) shows the behaviour of non-dimensional wall pressure distributions over the centrebody deduced from PSP. The pressure rise due to the SWBLI is ovelapping on top of each other for SWBLI@190, 200 and 220mm cases.SWBLI@90mm case has an abrupt rise whereas SWBLI@255mm case has a gradual behaviour. The locations for the pressure peaks are found to lie between 3 to $8 \mathrm{~mm}$ downstream of the inviscid position of the impinging shock. The most downstream position of the pressure peak is observed for SWBLI@255mm case.

\section{Conclusions}

An extensive experimental campaign was carried out to investigate transitional SWBLI at Mach 5 and $R e / m$ of $15.9 \cdot 10^{6} 1 / m$. An axisymmetric impinging shock that generates 7 degrees flow deflection, resulted in separated SWBLI flowfield on axisymmetric centrebody. Various flow diagnostics were utilised to map the complex flowfield such as schlieren photography, QIRT and PSP and pressure tappings. A nominally laminar (triggered transition) case by flow separation (SWBLI@90mm) and four natural transition cases with varying intermittency (SWBLI@190, 200, 

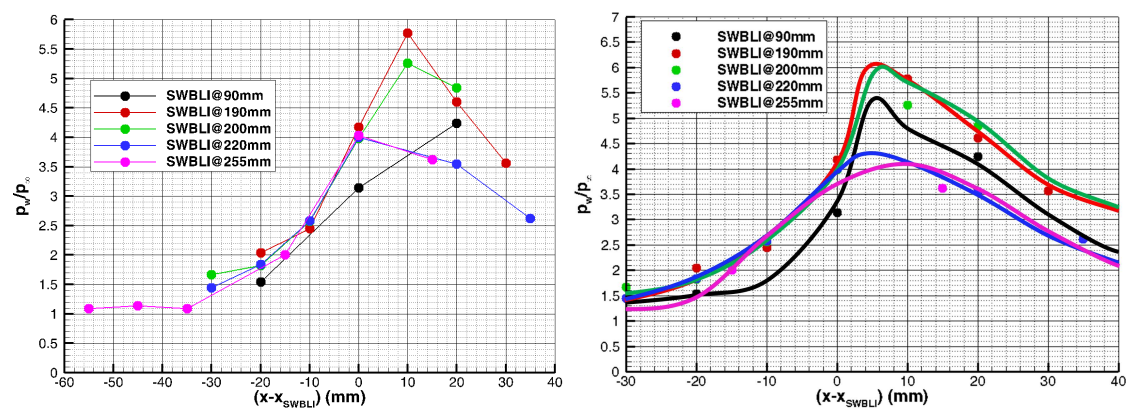

Fig. 5 Left: non-dimensional wall pressure distribution over the centrebody for all cases, right: non-dimensional wall pressure distribution over the centrebody deduced from PSP for all cases.

220 and $255 \mathrm{~mm}$ ) were tested. Time-averaged heat transfer peak was found to occur around the reattachment point. For SWBLI@90mm case the separation induces transition and the heat transfer and pressure peaks were found to be the highest. For the cases with natural transition with different intermittency levels, where incoming boundary layer is in state of transition, the magnitude of peaks initially started to increase from SWBLI@190mm case reaching a maximum for SWBLI@200mm case. Afterwards the peaks started decreasing towards the highest intermittency case, i.e. SWBLI@255mm.

\section{References}

1. Edney B: Anomalous heat transfer and pressure distributions on blunt bodies at hypersonic speeds in the presence of an impinging shock. Aeronautical Research Institute of Sweden, FFA Report 115, Stockholm, 1968

2. Delery J. and Marvin J.G.: Shock wave/Boundary Layer Interactions. AGARDograph No 280, 1986

3. Holden M.: A Review of Aerothermal Problems Associated With Hypersonic Flights. AIAA Paper 77-0045, 1986

4. Settles G. S.: Swept Shock/Boundary Layer Interaction Scaling Laws, Flow Field Structure and Experimental Methods. AGARD/FDP VKI Special Course on Shock Wave/Boundary Layer Interactions in Supersonic and Hypersonic Flows, AGARD Report No 792, 1993

5. Vandomme L., Chanetz B., Benay R. and Perraud J.: ShockWave/Transitional BoundaryLayer Interactions in Hypersonic Flow. AIAA J. 44(6): pp. 1243-1254, 2006

6. Boyce R. R. and Hillier R.: Shock-Induced Three-Dimensional Separation of an Axisymmetric Hypersonic Turbulent Boundary Layer. AIAA paper, 2000-2226

7. Murray N., Hillier R. and Williams S.: Experimental Investigation of Axisymmetric Hypersonic Shock-Wave/Turbulent-Boundary-Layer Interactions. J. Fluid Mech., Vol. 714, pp. 152189,2013

8. Erdem E. and Kontis K.: Numerical and Experimental Investigation of Transverse Injection Flows. J. Shock Waves, Vol. 20, No. 2, 103-118, 2010

9. Erdem E., Yang L. and Kontis K.: Steady Energy Deposition at Mach 5 for Drag Reduction. J. Shock Waves, Vol. 20, No. 2, 103-118, 2012 